\title{
Visualizing and Tracking Evolving Features in 3D Unstructured and Adaptive Datasets
}

Prof. D. Silver and Prof. N. Zabusky

GRANT NO. DE-FG02-98ER25364

Laboratory for Visiometrics and Modeling

CAIP Center, Rutgers University

P.O. Box 909, Piscataway, NJ 08855-0909

http://www.caip.rutgers.edu/vizlab.html

U.S. Department of Energy (DOE)

Chicago Field Office

9800 South Cass Avenue

Argonne, Illinois 60439

The material enclosed provides the final report of the Laboratory for Visiometrics and Modeling (Vizlab), CAIP Center, Rutgers University from DOE DE FG02 98ER25364 on the Grant entitled: Visualizing and Tracking Evolving Features in 3D Unstructured and Adaptive Datasets. 


\section{DISCLAIMER}

This report was prepared as an account of work sponsored by an agency of the United States Government. Neither the United States Government nor any agency Thereof, nor any of their employees, makes any warranty, express or implied, or assumes any legal liability or responsibility for the accuracy, completeness, or usefulness of any information, apparatus, product, or process disclosed, or represents that its use would not infringe privately owned rights. Reference herein to any specific commercial product, process, or service by trade name, trademark, manufacturer, or otherwise does not necessarily constitute or imply its endorsement, recommendation, or favoring by the United States Government or any agency thereof. The views and opinions of authors expressed herein do not necessarily state or reflect those of the United States Government or any agency thereof. 


\section{DISCLAIMER}

Portions of this document may be illegible in electronic image products. Images are produced from the best available original document. 


\section{Overview}

The massive amounts of time-varying datasets being generated demand new visualization and quantification techniques. Visualization alone is not sufficient. Without proper measurement information/computations real science cannot be done. Our efforts have always been to combine visualization with quantification of the data to allow for advanced querying and searching, which is the only way to handle massive databases to obtain new qualitative understanding of observed phenomena and to model quantitatively these observations. In our work, we show how quantification computations can improve the rendering and provide more meaningful visualizations without a significant cost in performance and processing time. Our focus has been on identifying and extracting features, quantifying and tracking them in time-varying simulations. Our research has been successful in presenting a new approach to the simulation and visualization community in visualizing data using feature tracking. This approach is of interest to DOE labs (Sandia, PPPL) and has been getting increased attention in the visualization conferences (by us and other researchers).

As part of this work we have used the projection of data to lower dimensions as a key to internalizing new ideas. This significantly reduces the amount of data to visualize and allows scientists to formulate reduced models to explain the behavior of coherent regions and their interaction with more diffuse statistical domains. Our research thrusts can be divided into two main areas, (a) Improving and enhancing feature extraction and tracking and $(b)$ examining new configurations and reduced modeling of accelerated inhomogeneous flows (AIFs).

\section{Overall Accomplishments 1998-2002}

\section{Visualization: Feature Extraction and Tracking}

- (1998-1999) We have completed the general implementation of feature extraction and tracking which operates on both structured and unstructured datasets. Our feature extraction and tracking environment is able to isolate thresholded regions from a 2D or 3D dataset and follow each region through all the timesteps, keeping track of various measurements as the feature evolves. The new program is general in that it uses linked lists and a merge-sort type algorithm to perform tracking. We have also significantly improved the run-time of the program from the previous version (Silver+Wang, 1997). The code operates under AVS 5. The unstructured and structured feature extraction and tracking code is available on our Web site http://www.caip.rutgers.edu/vizlab.html. The code available consists of AVS modules, Vis5D enhancements, Skeletal extraction modules, DX modules, and $\mathrm{C}++$ code.

- (1999-2002) We have designed and implemented a distributed version of the feature extraction code using MPI. Details of the algorithm are described in the next section. Three different algorithms were implemented. A distributed feature tracking program was also implemented. We have also begun implementing an AMR version of the algorithm to integrate fully with an ongoing simulation.

- (2000-2001) We have redesigned the user-interface, creating a stand alone interface written in Vtk to be more accessible to the Sandia Laboratories, and creating three new modes-of-operation within AVS and AVS express to make the code more accessible to both distributed and single processor implementation. Details are described in the next section. 
- (2002) We have made significant outreach efforts to Sandia Livermore and the Princeton Plasma Physics Laboratory and are now proceeding in porting our methodologies and codes for use in their environments. A recent paper by W. Koegler, Case Study: Applications of Feature Tracking to Analysis of Autoignition Simulation Data. Proceedings of IEEE Visualization '01, San Diego, CA, Oct 2001, utilizes our feature tracking approach for autoignition simulation data.

- (1998-2001) We developed a skeletonization algorithm to characterize 3D features (one of the many quantifications which are attached to the feature extraction process). The skeletonization algorithm has many other applications including reduced modeling, shape matching, shape registration and animation. We have used the skeletonization to characterize centerlines in hydrothermal plumes, to reshape volumetric datasets, to animate volumetric datasets, and to characterize volumetric shapes for future analysis.

\section{AIF \& Visiometrics}

- (1998-2001) We have identified the deposition of vorticity in single and double layers by various baroclinic processes including shock triple points and the emergence of vortex projectiles as the essential phenomena in AIF coherent structure, turbulence and species mixing evolution.. We have validated simulations by comparing with experiment and with other types of computer codes, including self-similar numerical solutions. Finally we are developing quantitative and reduced computer models that allow validation of simulations, robust prediction and design

- (1998-1999) Ray, Zabusky \& Samtaney modeled the deposition of vorticity on elliptic cylinders, including early breakthrough of transmitted shocks.

- (1999-2001) Ray, Kotelnikov, Gulak and Zabusky have modeled the early time growth of RM shock driven and ideal incompressible instabilities with compressible and incompressible codes. Comparisons with the dropped and reaccelerated tank gave good agreement. We introduced the concept of the time at which the interface becomes multivalued, as a prime quantification variable, and the width of the interface at this time.

- (1999-01) Samtaney and Zabusky developed an extraction technique for high gradient domains in 2D (shocks and contact layers) which uses a skeleton of the Lapalacian of density (numerical shadowgraph) or vorticity. Shocks are separated from contact layers by providing tests for pressure jumps. Samtaney has generalized this algorithm to $3 \mathrm{D}$, and we are in the process of using our 3D feature extraction and tracking techniques to analyze this data.

- (2000-01) Gupta, Zabusky, Gulak and Kotelnikov have developed a methodology for examining convergence of codes, particularly contact layer evolution (steepening or spreading). They performed a validation study of PPM and found unusual asymmetric spreading, which could prove trroublesome in reacceleration and reshock configurations.

- (2000-01) Peng and Zabusky have reexamined the high Mach number shock-bubble interaction in axisymmetry, in particular: modes of cavity collapse and reexpansion; formation of VPs and mass and circulation transport

- (2000-2001) S. Zhang, S. Gupta and G Peng and N. Zabusky have adapted two new EulerianLagrangian 3rd order hybrid algorithms which give results in excellent agreement for evolving discontinuous interfaces for a finite time in $2 \mathrm{D}$. Zhang has begun converting the code to use AMR 
data structures and run in the GrACE parallel/distributed environment (see next section). This code will be integrated with the AMR feature extraction code to demonstrate a computational steering environment that relies on reduced-data-visualization to achieve comprehension and interactivity.

\section{Research Accomplishments}

\section{Distributed Processing}

We have completed a distributed (parallel) version of the feature extraction process so that massive datasets $\left(>256^{3}\right)$ can be tracked without running into the usual bottleneck of the dataset being too large too fit onto a single processor. The code runs on both structured and unstructured datasets and outputs directly into the feature tracking code to correlate the features from different timesteps. An overview of the distributed code is shown in Figure 1. Two different implementation of the algorithm were developed. In the first, each processor extracts their own features and then a binary swap algorithm takes over to merge neighboring features. Because features can potentially span over all of the processor, an O(nlogn) communication-laden algorithm results. The second algorithm is called a "Partial Merge Strategy". In this algorithm, after each processor does its own feature extraction, only neighboring regions compute the merge (i.e., using ghosted boundaries). This partial connectivity is then sent along with the resulting features (or boundary triangles) to the visualization server. The visualization server computes the global merge. That information can then be transmitted a visualization program (either within AVS or the VTK interface, see below) for feature tracking and rendering. The code is now being written using GRaCE for AMR simulations (http://www.caip.rutgers.edu/ parashar).

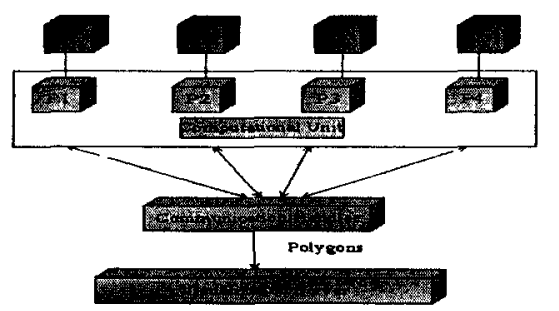

Figure 1: Distributed Feature extraction algorithm

\section{User Interface}

We have redesigned the interface to the feature extraction and tracking program to accommodate distributed processing. There are two new interfaces, one based upon AVS and the other on Vtk. An overview of the feature tracking environment is shown in Figure 2.

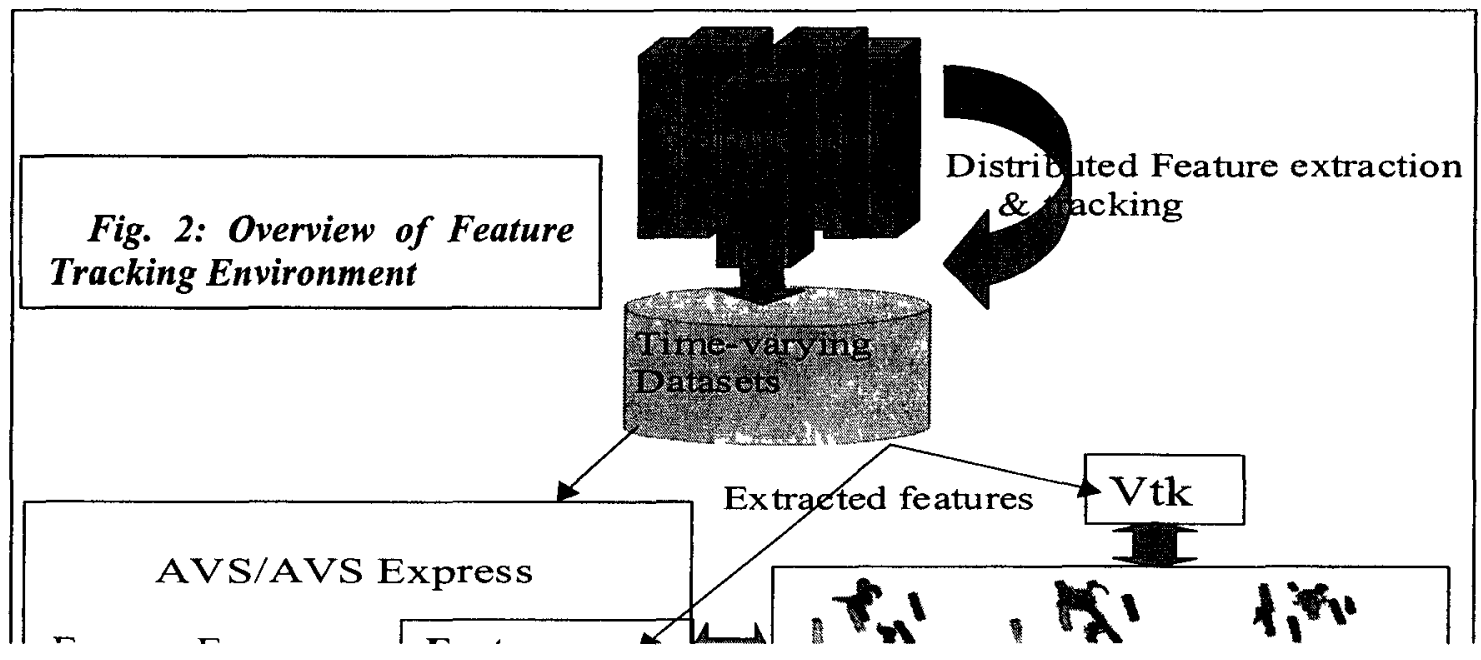




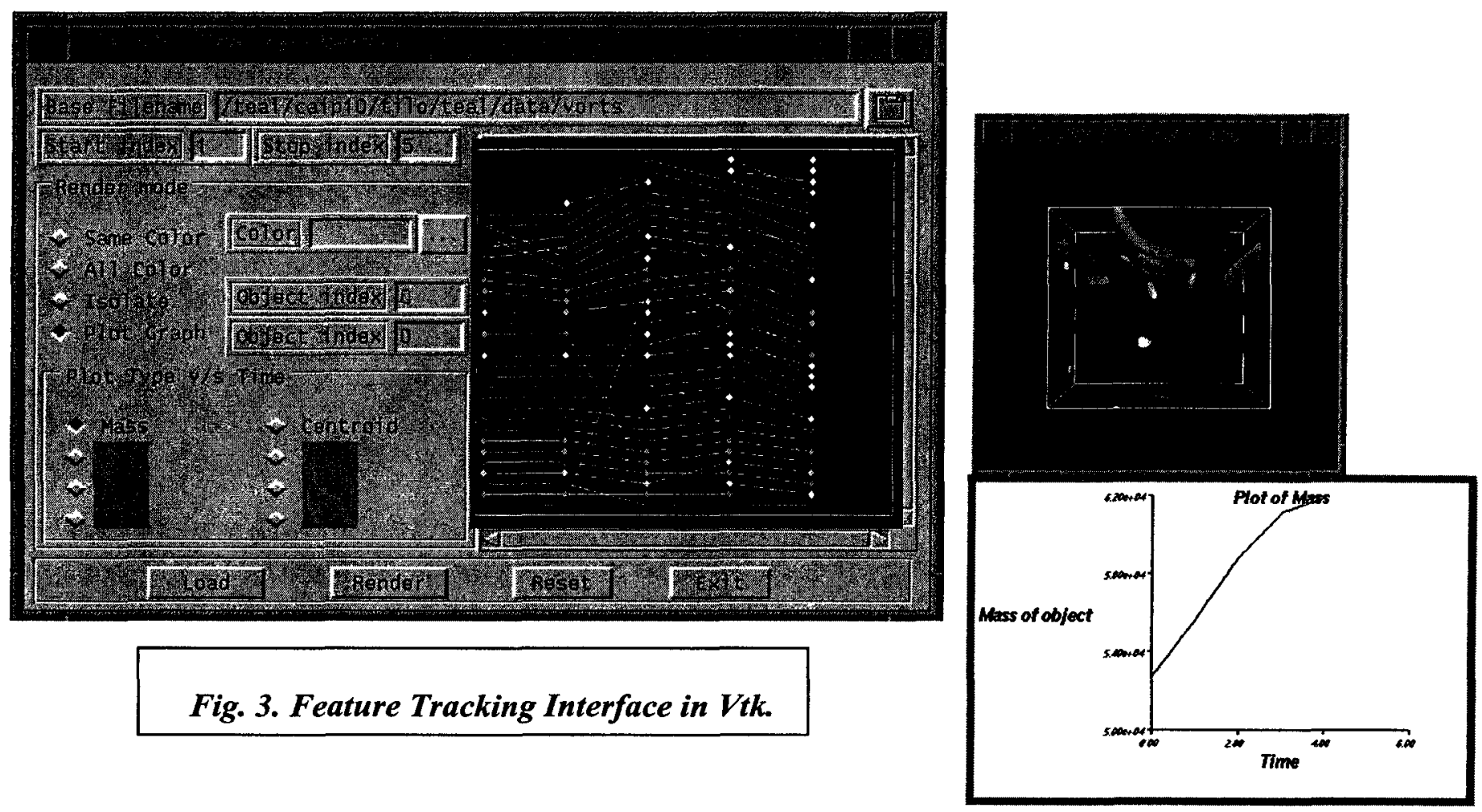

The original feature extraction and tracking program and visualization thereof was written within AVS 5. We have now decoupled the interface into three separate components: Feature extraction (with its own interface), Feature Tracking and Visualization. A new interface was written within Vtk (which is shown in Figure 3). This new interface can be used by a distributed simulation+tracking program to view the reduced data results. The Vtk feature visualizer is tailored to tracking and allows the user to select features, view their time histories, and define quantifications (e.g. mass).

\section{Skeletons}

We have made improvements in characterizing the extracted domain using skeletons. The skeletons are connected thin versions of a feature which capture the essential shape of the object (centerlines). They are computed in the volume domain (unlike other geometric algorithms) and therefore do not suffer from roundoff error. Our algorithm is robust and semi-reversible and has many other applications such as shape matching, shape registration, reduced modeling and volume animation. We are currently working with oceanographers to use the centerline to model hydrothermal plumes.

\section{Accelerated Inhomogenous Flows}

In the area of AIF we are particularly interested in the Richtmyer-Meshkov and Rayleigh-Taylor instability. Our major geometries for simulation are inclined curtain and axisymetric bubble. To direct our researches toward 3D, high-resolution, physically complex and numerically intensive problems, we integrated the dynamic AMR engine GrACE with the high-order-of-accuracy numerical computation scheme (Piecewise Parobolic Method). We have run simulations with different levels of refinement and on different numbers of processors. The results so far show good correspondence with our previous results using a sequential, uniform grid scheme. We have improved our computational time and hope to be producing large 3D datasets by the end of the summer. 


\section{Outreach}

D. Silver gave presentations on Feature Extraction and Tracking at Sandia New Mexico (contacts: D. Pavlakos and P. Crossno) (December 2000) and at Sandia Livermore (contacts: W. Koegler and R. Armstrong) (January 2001). She has also given talks at the PPPL. The Reacting Flow Research Group at Sandia Livermore, and the visualization group at Princeton Plasma Physics Laboratory are interested in incorporating the Feature Tracking into their visualization efforts. We are actively working with $\mathrm{W}$. Koegler at Sandia and S. Klasky at PPPL to achieve this integration and are currently preparing a next phase of this grant to deploy the code in a DOE environment.

N. Zabusky has given many presentations at Universities, Research Institutes, Physics and AIAA Meetings and Specialized meetings (e.g. IUTAM, ICTAM, IWPCTM, IWCCPA, etc). In addition, he was invited for an extended visit at the Institute of Laser Engineering (ILE,(2/1/01-6/30/01)) in Osaka, Japan. The collaboration there consisted of six lecture short course and research with Prof. K. Nishihara, students and colleagues. (ILE is engaged in the fundamentals and applications of ICF). Based on our success with the ILE group we are beginning to reinitiate contact with the comparable PPPL, LANL and LLNL groups

\section{Areas for Future Research Efforts}

We are currently preparing a continuation proposal to expand on the work we have done so far. Some of the areas of future efforts include distributed tracking program, a fully integrated AIF simulation and tracking environment using AMR, and integrating our feature extraction and tracking environment within the DOE laboratories.

\section{Recent Talks and Publications}

J. Chen, D. Silver and Y. Kusurkar, Distributed Feature Extraction and Tracking, SPIE IS\&T Electronic Imaging Symposium, Visualization and Data Analysis Conference, January 2002, San Jose, CA.

Y. Kusurkar, Distributed Feature Extraction and Tracking, MS. Thesis, Department of Electrical and Computer Engineering, Rutgers University, September 2001.

Visualizing Time Varying Phenomena", Sandia National Laboratories, D. Pavlakos and P. Crossno hosts, Albuquerque, New Mexico December 2000.

"Volume Animation", Department of Computer Science, University of New Mexico, December 2000

"Visualizing Time Varying Phenomena", Sandia National Laboratories, Computational Modeling and Simulation Group, (W. Koegler and R. Armstrong hosts), Livermore, California, January 2001

"Volume Animation \& Feature Tracking", TU Delft, The Netherlands, March 2001.

D. Silver and Y. Kusurkar, Visualizing Time Varying Distributed Datasets, VDE2000 Proceedings, Princeton Plasma Physics Laboratory, Princeton NJ, April 2000.

Feature Extraction and Tracking, Springschool lecture course, University of Bonn,.Germany, March 2000.

Parameter Controlled Volume Thinning, N. Gagvani and D. Silver, Graphical Models and Image Processing, Academic Press, p 149-164, vol 61 number 3, 1999. 
Flow Visualization, Wiley Encyclopedia of Electrical and Electronics Engineering, Volume 7, 1999. ed J. Webster.

Visualizing Evolving Scalar Phenomena, X. Wang and D. Silver, Journal of Future Generation Computer Systems (FGCS), Elsevier, Volume 15, Number 1, p 99-108, 1999.

Feature Extraction and Tracking, IEEE Visualization 1999, course notes, October 1999.

"Scientific Computing Visualization - A new venue in the arts",I Plenary session talk. N. Zabusky, Science and Art Symposium 2000, Third Int. Conference, Zurich, Switzerland, March 2000.Kluwer 2000, pp1 -19 .

"Accelerated Inhomogeneous Flows" and "Vorticity Deposition and Evolution by Shock Waves", N. Zabusky, Hydrodynamical and Astrophysical Turbulence Workshops, Inst of Theoretical Physics, Santa Barbara, CA. May 2000.

AIAA paper 2000-2412. "Visiometrics for reduced modeling of accelerated inhomogeneous flows." Fluids 2000, Denver, CO, 6/20/00.

International Congress of Theoretical \& Applied Mechanics (ICTAM 2000), Chicago, Il. Aug $27-$ Sept. 2. "Nonlinear Evolution and Vortex Localization in Richtmyer-Meshkov Accelerated Interfaces". N. J. Zabusky, A. D. Kotelnikov, and Y. F. Gulak

Florida State University, Center for Computational Science, "Nonlinear Evolution and Vortex Localization in Richtmyer-Meshkov Accelerated Interfaces“. Oct 19, 2000

Institute of Laser Engineering, Osaka University, Osaka, Japan. April, May 2001. Six seminar short course on: "Accelerated Inhomogeneous and Vortex Dominated Flows: Visiometrics and Modeling".

Earth Simulator Research \& Development Center in Yokohama, Japan. April 24,2001 "IT Aspects of Visiometrics \& Modeling in Computational Science: From the soliton to fusion"

International Workshop on Computational Methods for Continuum Physics \& Their Applications. (IWCCPA) Nanjing, China, May 22, 2001 (plenary session) "Vortex Double Layers and Vortex Projectiles in Accelerated Inhomogeneous Flows"

High-Gradient compressible flows: Visualization, feature extraction and quantification in Flow visualization, techniques and examples". R. Samtaney and N.J. Zabusky. 2000. Eds A. Smits and T. Lim. Imperial College Press.pp317-344 and color plates.

Vortex paradigm for accelerated inhomogeneous flows: Visiometrics for the Rayleigh-Taylor and Richtmyer-Meshkov environments. N.J. Zabusky. Annual Review of Fluid Mechanics, 1999. 31:495535.

Visualization, Feature Extraction and Quantification of Numerical Simulations of High Gradient Compressible Flows, R. Samtaney and N. Zabusky. Imperial College Press, A. Smits and T. Lim, Eds, 2000 .

Shock Interaction with Heavy Gaseous Elliptic Cylinders: Two Leeward Side Shcok Competition Modes and a Heuristic Model for Interfacial Circulation Deposition at Early Times, J. Ray R. Samtaney and N. J. Zabusky, Phys. Flds, 12(3):707-716, 2000. 
Vortex Dynamics of a Twice-accelerated Interface in an Incompressible Ideal Fluid", A. Kotelnikov and N. Zabuksy, The Astrophysical Journal Supplement Series, 127, April 2000.

Vortex morphologies on reaccelerated interfaces: Visualization, quantification and modeling of one-andtwo mode compressible and incompressible environments. A. Kotelnikov, J. Ray, and N.J. Zabusky. Physics of Fluids 12, Dec 2000,

Nonlinear Evolution and Vortex Localization: Different Phases of a Single Mode Richtmyer-Meshkov Unstable Interface. A. Kotelnikov, Y. Gulak and N.J. Zabusky. Submitted to Journal of Fluid Mechanics January, 2000.Accepted subject to revision

Shock-planar curtain interactions: Strong secondary baroclinic deposition and the emergence of coherent and vortex projectiles (VPs) and decaying stratified turbulence. S. Zhang and N. J. Zabusky, Physics of Fluids . Accepted subject to revision, April 2001

A Novel Experiment on the Blast Wave-Sphere Interaction Using Laser Produced Plasma.Y.-G. Kang,

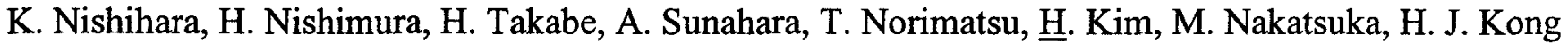
and N. J. Zabusky. Phys Rev E, submitted May 2001

\section{Personnel (relating to Vizlab efforts)}

Dr. J. Ray received his $\mathrm{PhD}$ in MAE from RU in May 1999. His research emphasized simulation and quantification of AIFs. He received a Postdoc at Sandia Livermore in the Combustion research area, and he has a permanent job there.

Dr. A. Kotelnikov, PostDoc, Sept 1999-Sept 2000 (PhD Dartmouth under D. Montgomery) continued research on accelerated inhomogeneous flows (AIFs),emphasizing simulation and juxtaposition with experiments.

Dr. Y. Gulak, Postdoc May 1999-May 2000 (PhD Rutgers, under R. Pelz) continued his research on AIFs emphasizing computer algebra solutions, validation and predictability of algorithms and codes. Dr. H. Ray received his Ph.D. in ECE from RU (2000). His research emphasized a new hardware architecture for real-time volume rendering. He is now working at Hewlett Packard.

Dr. X. Wang received his Ph.D. from the Dept. of Electrical and Computer Engineering in 1998. His research was in feature extraction and Tracking. Dr. N. Gagvani received his Ph.D. from the Dept. of Electrical and Computer Engineering in January 2001. His research work involved skeletonization.

C. Feng has received his MS degree in Feature Extraction and Tracking (December 1999); Y. Kusurkar (distributed feature extraction in September 2000) and K. Sen (skeletonization, June 2001). $W$. Townsend and D. Deleon received their BS degrees (1999), and A. Martinez received his BS degree in 2001. They were undergraduate researchers in the Vizlab. T. Roy (AVS volume tracking, August 2002) and A. Battacharya (Skeletonization, August 2002) also received their MS. Degrees.

Prof. N. J. Zabusky received a Jacobs Chair in Applied Physics from Rutgers for three years, from 1/1/00-12/31/02. Besides the honor, the funds will allow: support of one GRA; support of research in data projection as part of the DAVID environment; visits to RU from collaborating and distinguished scientists; visits to meetings and institutes in Japan, China and Poland; and ability to explore new areas, (e.g. AIFs and computational astrophysics; computers, color maps and art; and history of computational physics in the 20th century, etc). 